\title{
Agencia personal y empoderamiento en beneficiarias del programa juntos, Ayacucho
}

\author{
Personal agency - empowerment in beneficiaries of program juntos, \\ Ayacucho \\ Betty García Vargas ${ }^{1}$ \\ Ministerio Público \\ Alberto Loharte Quintana Peña ${ }^{2}$ \\ Universidad Nacional Mayor de San Marcos
}

Recibido: $04-10-19$

Aceptado: $17-12-19$

\section{Resumen}

El presente estudio tuvo como objetivo identificar el nivel de agencia personal y empoderamiento en beneficiarias del programa Juntos del distrito y provincia de Vilcashuamán del departamento de Ayacucho. La investigación es de diseño descriptivo comparativo. La muestra conformada por 307 beneficiarias se dividió en dos grupos, 143 beneficiarias con menos de 2 años de participación y 164 beneficiarias con más de 3 años de participación en el programa Juntos. El instrumento utilizado corresponde a la escala de Agencia Personal y empoderamiento de Pick et. al. (2007). En los resultados se encontró que la capacidad de agencia personal es mayor en el grupo con 3 años a más que el grupo de 2 años a menos dentro del programa Juntos. En tanto que el nivel de empoderamiento no mostró mayor variación de un grupo a otro. Se sugiere reproducir el estudio a fin de profundizar y analizar las variables en este tipo de población.

Palabras clave: Agencia personal; empoderamiento; programa Juntos.

\begin{abstract}
The present study aimed identify the level of personal agency and empowerment in the beneficiaries of the program Juntos from Vilcashuaman - Ayacucho. The research is of descriptive comparative design. The sample consisted of 320 beneficiary between which have less two years and more three years into the program Juntos. The instrument was Personal Agency and Empowerment Scale of Pick (2007). The results concluded that personal agency increase according the time and empowerment shows no difference from one group to another. Research is recommended to reproduce in order to deepen and analyze the features involved in this type of population.
\end{abstract}

Keywords: Personal Agency; empowerment; and Programa Juntos.

1 Psicóloga en Ministerio Público. E-mail: annybetgv@gmail.com

2 Docente principal, Universidad Nacional Mayor de San Marcos. E-mail: aquintanap@unmsm.edu.pe

(C) Los autores. Este artículo es publicado por la Revista de Investigación en Psicología de la Facultad de Psicología, Universidad Nacional Mayor de San Marcos. Este es un artículo de acceso abierto, distribuido bajo los términos de la licencia Creative Commons Atribucion - No Comercia_Compartir Igual 4.0 Internacional. (http://creativecommons.org/licenses/by-nc-sa/4.0/) que permite el uso no comercial, distribución y reproducción en cualquier medio, siempre que la obra original sea debidamente citada. 
El departamento de Ayacucho es uno de las regiones andinas que ha sufrido y sigue padeciendo los estragos de la violencia política desatada en la década de los $80^{\prime}$ a manos de grupos terroristas y paramilitares. A estos se suma la serie de sucesos económicos que generaron un escenario de hiperinflación a finales de la década. En este contexto se promueven el surgimiento de programas sociales como son el vaso de leche y programas alimentarios. Para la década de los $90^{\prime}$ debido a la crisis económica estos programas se distorsionan por intereses políticos. Con la caída del gobierno de turno surge la necesidad de replantear los programas sociales que a su vez coincide con la declaración del milenio de las naciones unidas, donde se determina una serie de objetivos respecto del bienestar humano para ser alcanzados al 2015. Es así que en el año 2005 se da inicio al programa de transferencia monetaria conocido como JUNTOS, que inicia operaciones en el distrito de Chuschi - Ayacucho, para progresivamente ampliarse a todos los departamentos del Perú.

El Programa Nacional de Apoyo Directo a los más Pobres - JUNTOS en el Perú (Programa de Transferencias Monetarias Condicionadas) - incluida en los lineamientos político sociales de lucha para combatir la pobreza del estado Peruano, bajo la estrategia CRECER- cuyo objetivo es erradicar la pobreza y generar capital humano a través de la transferencia de recursos económicos para promover la concurrencia a las áreas de salud, nutrición, educación e identidad, y así frenar la transmisión intergeneracional de la pobreza extrema. Este aporte monetario se supedita a una serie de compromisos que asume cumplir el hogar beneficiario, buscando así promover y garantizar el acceso y participación de los hogares pobres con gestantes y niños menores de 14 años en las áreas mencionadas, fomentando la corresponsabilidad con el estado. Dicho incentivo es entregado a las madres de familia, considerada por el Programa como titulares del hogar beneficiario. Al ser las mujeres, las administradoras directas, se mejora su participación económica dentro del hogar, reflejándose según estudios la mejora de empoderamiento en el ámbito familiar (Instituto de estudios peruanos, 2009). El presente estudio se basa en este Programa JUNTOS el cual señala como objetivo promover el desarrollo humano, observándose que para conseguirlo se desarrollan variables psicosociales como la agencia y empoderamiento.

La agencia personal como lo entiende Amartya Sen, es la habilidad de definir las metas propias de forma autónoma y de actuar a partir de las mismas: "es aquello que una persona tiene la libertad de hacer y lograr en la búsqueda de las metas o valores que él o ella considera importantes" (citado en Pick et. al. 2007). "Es la capacidad para actuar de manera autónoma" (Padilla, 2011). Promover la agencia personal eleva la probabilidad de actuar y motivar cambios, conllevando a mejorar la calidad de vida de las personas, promoviendo que se ayuden a sí mismos e influyan en su contexto. Para Ibrahim y Alkire (2007) "la agencia es necesaria para el empoderamiento e incrementarlo implica a su vez incrementar 
la agencia, aunque agencia no necesariamente implica empoderamiento" (como se cita en Valladares, 2011). Así pues cuando la agencia empieza a impactar sobre la familia, colegas, organizaciones y comunidad, se transforma en empoderamiento. Entendiendo el empoderamiento como la capacidad para ejercer efectiva y consecuentemente los derechos e incrementar el protagonismo en la vida pública (Instituto de estudios peruanos, 2009); autores como Pick et.al. (2007) definen el empoderamiento como la expansión de la agencia, Drydyk (2008) señala que no se trata de cualquier tipo de agencia, sino de aquella que incide sobre el bienestar y en el logro de objetivos que las personas valoran (como se cita en Valladares, 2011)

Entre las investigaciones sobre agencia y empoderamiento se puede mencionar a Pick (2011), que concluyó que a través del desarrollo de conocimientos y habilidades se fomenta sobre el desarrollo de la agencia personal en habitantes de comunidades marginadas. Del mismo modo Matijasevic (2010) halló que en habitantes de Manizales - Colombia, la capacidad de agencia denota una alta valoración para procurarse propósitos valiosos en sus vidas, sin desconocer que existen restricciones; lo cual guarda concordancia con su percepción de bienestar sobre su estado de salud, vivienda y logros alcanzados, aspectos que están dentro de su control, en tanto que aspectos como el trabajo, vida económica y educación su percepción de bienestar es menor.

En el ámbito nacional Padilla (2011) en el contexto de microempresa describe que a mayor agencia personal la percepción de rol de género en las mujeres es menos estereotipada, es decir que la oportunidad de trabajar y de ser independiente económicamente coadyuva al crecimiento de agencia personal y a poner en debate y cuestionar los roles estereotipados de la mujer, asignándole poder ser asertiva y líder, características habitualmente atribuidas a los varones. Por otro lado Barbier, (2014), encontró diferencias significativas en la Agencia personal y Empoderamiento según grado de instrucción, estableciendo que la oportunidad de educación facilita y promueve el desarrollo de la capacidad agéntica y empoderamiento.

Así pues estas variables psicosociales, ofrecen oportunidades para superar la pobreza (Pick, García. \& Leenen, 2011) de ahí que surge el interés por conocer el comportamiento de dichas variables sobre esta población en específico, pues las investigaciones realizada al respecto de estas variables en esta población son escasas si no nulas.

El presente estudio pretende conocer los niveles de agencia personalempoderamiento en las beneficiarias del programa juntos y cómo dichas variables se desarrollan en función del tiempo de participación en el programa; así pues sí, la tendencia es el incremento a través del tiempo se podría presumir que las personas involucradas mantendrían conductas favorables a su desarrollo aun cuando el programa social dejará de existir. 


\section{MÉTODO}

\section{Tipo y diseño de Investigación}

La investigación es de corte descriptivo comparativo, descriptivo pues muestra las variables de estudio en dos grupos que difieren en su tiempo de participación en un programa social del estado; y es comparativo dado que se establece "relaciones entre las variables de estudio sin precisar sentido de causalidad”. (Hernández et al., 2010).

\section{Muestra}

La población conformada por las beneficiarias del Programa Juntos, pertenecientes al distrito de Vilcas Huamán, Provincia de Vilcas Huamán, Departamento de Ayacucho. El muestreo es seleccionado por el mismo investigador, acorde a su criterio considerando la representatividad -por tanto intencional-, no probabilístico. La muestra fue de 307 mujeres adultas, madres de familia, entre los 20 y 50 años de edad, cuya primera o segunda lengua es el castellano y que son beneficiarias del Programa Juntos, del distrito de Vilcas Huamán, Provincia de Vilcas Huamán, Departamento de Ayacucho. La muestra compuesta por un $47 \%$ (143) de beneficiarias con dos años a menos de participación en el programa Juntos y un 53\% (157) de beneficiarias con más de dos años de participación en el programa.

\section{Instrumento}

La Escala para medir la Agencia Personal y Empoderamiento (ESAGE) elaborado por el Instituto Mexicano de Investigación de Familia y Población (IMIFAP) dentro del marco de "las evaluaciones de Proceso e impacto de programas de salud, equidad de género y desarrollo económico realizados en México y países de Centro y Sudamérica" (Pick et al., 2007). Conformado por 42 reactivos, agrupados en dos dimensiones: agencia personal (35 reactivos) y empoderamiento (7 reactivos) ítems, es autoaplicable y se responde con una escala tipo Likert. En la construcción del instrumento, se confirmó que los dos factores estaban ligeramente correlacionados de modo significativo $(r=-$ $0.131, p<0.01)$. Seguidamente, se midió la confiabilidad de dichos factores, estableciendo su Alfa de Cronbach, hallándose en los dos factores un elevado nivel de consistencia entre los reactivos (Agencia, $a=0.718$; Empoderamiento, $a=0.749$ ). La evaluación determinó que la ESAGE posibilita evaluar con adecuada validez y confiabilidad el impacto generado por ambos factores: la agencia personal y el empoderamiento, además permitió confirmar que son dos factores relacionados entre sí y a la vez independientes.

Para la presente investigación, si bien la ESAGE posibilita evaluar con adecuada validez y confiabilidad la agencia personal y empoderamiento, se observó que posee ítems cuyos coeficientes ítem-total arrojan valores inferiores 
a 0,35, valor considerado estadísticamente significativo (Cohen-Manion, 1990). Concluyendo así, descartar aquellos ítems cuyo valor no supera el valor indicado. Por tanto la presente investigación, empleó una ESAGE con 33 reactivos, 27 ítems para agencia personal y 6 ítems para Empoderamiento. Finalmente se solicitó a 5 jueces conocedores del tema revisar cada ítem para así validar su eficacia en la medición del constructo, como su forma lingüística. Posterior a la aplicación del instrumento, se realizó un análisis de confiabilidad a través del método de consistencia interna y empleando el coeficiente alfa de Cronbach (Kerlinger \& Lee, 2002). Para agencia personal se obtuvo un alfa de Cronbach de 0.608 nivel considerado bajo pero admisible según Murphy y Davishofer, (1998) (citado en Hogan, 2004); a nivel de ítem-test se obtuvo que 16 de los 27 ítem tenían una correlación inferior a 0.20 , dado que la eliminación de estos ítems elevaba sensiblemente la confiabilidad del instrumento, se consideró no emplearlos en análisis posteriores. En cuanto la dimensión de empoderamiento se observa un coeficiente alfa de Cronbach de 0.598, para Murphy y Davishofer (1998) es un nivel considerado inaceptable (citado en Hogan, 2004). Dicho nivel se explica por los índices de correlaciones ítem-test ya que de los 6 ítems solo uno no alcanzó la correlación superior a 0.20 . Posterior a la depuración de un ítem, se hizo un análisis final de confiabilidad con los reactivos restantes. El nuevo coeficiente alfa de Cronbach fue igual a 0.676, nivel considerado bajo pero aceptable para Murphy y Davishofer (1998) (citado en Hogan, 2004).

\section{Recojo y análisis de información}

Se realizó las gestiones iniciales con la promotora del programa Juntos de la zona a intervenir, acordando fecha y lugar para convocar a las beneficiarias y proceder a la aplicación colectiva de la escala, no sin antes obtener autorización mediante consentimiento informado. La información fue procesada a través del programa Statistical Package for the Social Sciences (SPSS-18). En lo descriptivo, se empleó tablas de frecuencias y porcentajes de las variables interdependientes y del tiempo de permanencia de las beneficiarias en el programa JUNTOS. A nivel inferencial, se realizó un análisis comparativo de las variables interdependientes con las variables de control según las hipótesis planteadas, para lo cual se utilizó el estadístico paramétrico T de Student para muestras independientes.

\section{RESULTADOS}

\section{Análisis descriptivo}

Se presentan los resultados descriptivos sobre la variable agencia personal, empoderamiento y la variable de permanencia en el programa Juntos. 
La Tabla 1 permite apreciar que la dimensión de Agencia Personal en las beneficiarias de dos años o menos como en las de tres años o más obtiene valores de $69 \%$ y $64 \%$ respectivamente, categorizando en un nivel medio. Adicionalmente, se aprecia que las beneficiarias con dos años o menos en el Programa Juntos tienen una tendencia baja (18\%) de Agencia Personal; por el contrario, las beneficiarias con tres años o más poseen una tendencia alta (21\%).

\section{Tabla 1}

Resultados descriptivos de la dimensión de Agencia Personal en beneficiarias del Programa Juntos según tiempo de permanencia $(n=307)$

\begin{tabular}{lccccc}
\hline & & \multicolumn{2}{c}{ Dos años o menos $(\mathbf{n}=\mathbf{1 4 3})$} & \multicolumn{2}{c}{ Tres años o más $(\mathbf{n}=\mathbf{1 6 4})$} \\
\cline { 3 - 6 } & & $\mathbf{f}$ & $\mathbf{\%}$ & $\mathbf{f}$ & $\mathbf{\%}$ \\
\hline \multirow{3}{*}{ Agencia } & Alta & 19 & 13 & 34 & 21 \\
Personal & Media & 98 & 69 & 105 & 64 \\
& Baja & 26 & 18 & 25 & 15 \\
\hline Total & & 143 & 100 & 164 & 100 \\
\hline
\end{tabular}

Dentro de la Tabla 2 se observa que la dimensión Empoderamiento tanto en las beneficiarias con dos años o menos y de las de tres años o más lograron niveles medios de $73 \%$, en ambos casos. Así mismo se aprecia que para el caso de las beneficiarias con dos años o menos hay una tendencia alta (15\%); sin embargo, para las beneficiarias con tres años o más no hay una tendencia clara y se mantiene en un nivel de empoderamiento medio.

\section{Tabla 2}

Resultados descriptivos de la dimensión de Empoderamiento en beneficiarias del Programa Juntos según tiempo de permanencia $(n=307)$

\begin{tabular}{lccccc}
\hline & & \multicolumn{2}{c}{ Dos años o menos $(\mathbf{n}=\mathbf{1 4 3})$} & \multicolumn{2}{c}{ Tres años o más $(\mathbf{n = 1 6 4})$} \\
\hline \multirow{3}{*}{ Empoderamiento } & $\mathbf{f}$ & $\mathbf{\%}$ & $\mathbf{f}$ & $\%$ \\
& Alta & 21 & 15 & 22 & 13 \\
& Media & 104 & 73 & 119 & 73 \\
& Baja & 18 & 12 & 23 & 14 \\
& Total & 143 & 100 & 164 & 100 \\
\hline
\end{tabular}

\section{Análisis inferencial}

La Tabla 3 muestra los estadísticos de las variables de estudio. En primer lugar, la variable Agencia Personal tiene una media 24.24 y una variación de 6.14; en la variable Empoderamiento hay un promedio de 15.62 y una variabilidad de 3.10. En la prueba de normalidad Kolmogorov-Smirnov se establece que las variables de estudio alcanzaron un p-valor superior al 0.05 ; por tanto, los datos provienen de 
una población de distribución normal. En tal sentido, se hizo usó de la estadística de tipo paramétrica para el análisis inferencial.

Tabla 3

Estadísticos y prueba de Normalidad de las variables (Agencia Personal y Empoderamiento) en beneficiarias del Programa Juntos ( $n=307$ )

\begin{tabular}{lcccc}
\hline Variable & $\begin{array}{c}\text { Media } \\
\text { Aritmética }\end{array}$ & $\begin{array}{c}\text { Desviación } \\
\text { Estándar }\end{array}$ & $\begin{array}{c}\text { Kolmogorov- } \\
\text { Smirnov }\end{array}$ & $\begin{array}{c}\text { p-valor } \\
\text { (bilateral) }\end{array}$ \\
\hline Agencia Personal & 24.24 & 6.14 & .108 & $.000^{* *}$ \\
Empoderamiento & 15.62 & 3.10 & .106 & $.000^{* *}$ \\
\hline
\end{tabular}

$* \mathrm{p}<.05 ; * \mathrm{p}<.01$

\section{Análisis comparativo}

Para el respectivo análisis se empleó la prueba paramétrica $T$ de Student para muestras independientes. La Tabla 4, muestra diferencias significativas entre ambos grupos (dos años o menos y tres años o más), a favor, del grupo de tres años o más. En cuanto la dimensión Empoderamiento no se hallaron diferencias significativas entre ambos grupos.

\section{Tabla 4}

Prueba t de Student para muestras independientes para estimar si existen diferencias entre las dimensiones Agencia Personal y Empoderamiento según tiempo de permanencia $(n=307)$

\begin{tabular}{cllccc}
\hline Dimensión & \multicolumn{1}{c}{ Grupo } & N & Media & t & gl (p-valor) \\
\hline Agencia Personal & Dos años o menos & 143 & 23.08 & -3.135 & 300.874 \\
& Tres años o más & 164 & 25.24 & & $(.002)^{* *}$ \\
\hline Empoderamiento & Dos años o menos & 143 & 15.87 & 1.360 & 289.450 \\
& Tres años o más & 164 & 15.39 & & $(.175)$ \\
\hline
\end{tabular}

Nota: $\mathrm{t}$, coeficiente t de Student; gl, grados de libertad; ${ }^{*} \mathrm{p}<.05 ; * * \mathrm{p}<.01$

\section{DISCUSIÓN}

El nivel de agencia personal muestra una diferencia significativa entre el grupo de beneficiarias con 2 años a menos y el grupo de beneficiarias con 3 años a más de participación en el programa Juntos, siendo la tendencia favorable al grupo de 3 años a más. Lo encontrado es compatible con el estudio que realizó Pick; García \& Leenen (2011) en donde tras aplicar un Programa para modificar conductas a través del desarrollo de conocimientos y habilidades psicosociales lograron un efecto positivo sobre la agencia personal entre otras habilidades más en habitantes de comunidades marginadas. Así mismo Perova y Vakis (2011) encontraron que los efectos del Programa Juntos respecto a la utilización de servicios de salud y educación son significativamente más altas luego de pasar 
12 meses en el programa. Tal como Matijasevic (2010) observó en habitantes de Manizales - Colombia, donde la capacidad de agencia denota una alta valoración para procurarse propósitos valiosos en sus vidas, es así que su percepción de bienestar sobre su estado de salud, vivienda y logros alcanzados, es mayor ante aspectos como el trabajo, vida económica y educación. Es de esperarse entonces que su capacidad agéntica se vea incrementada conforme permanece más tiempo en el programa Juntos.

Respecto al nivel de empoderamiento no se aprecia diferencia entre ambos grupos. Si bien Drydyk (2008) describe el empoderamiento como la expansión de la agencia (citado en S. Pick, 2011); Ibraham y Alkire (2007) refieren que empoderamiento implica que incremente la agencia; pero agencia no necesariamente implica empoderamiento. Así también Pick et. al. (2007) señala que el proceso interno de adquirir control es la agencia, y el impacto de la agencia a nivel externo y los cambios en su comunidad, se denomina empoderamiento. El cual tiene que pasar por una serie de etapas para conseguir cada vez más agencia, reflejándose en acciones valiosas y significativas que repercuten en su contexto y trascendiendo a un nivel comunitario más amplio. El que la agencia no logre concretarse en empoderamiento aun cuando se aprecia incremento de la capacidad agéntica, podría deberse a un tema de limitaciones asociadas al género y a la vulnerabilidad económica (Padilla, 2011). Pese a que las beneficiarias tienen mayor protagonismo en la administración del dinero recibido -factor que influye para expandir la agencia y conllevar a empoderamiento (Zambrano, 2007, citado en Padilla, 2011) - no significa incremento en su nivel de autoconfianza para la toma de decisiones y realizar cambios de manera autónoma en su entorno. Es así que la muestra del estudio, conformada por mujeres beneficiarias residentes de una zona altoandina del departamento de Ayacucho, posee arraigados aún los estereotipos de su género y que se desarrollan en un contexto socio cultural donde el machismo aún subsiste; es de comprender entonces que no haya diferencia significativa en cuanto al empoderamiento.

\section{CONCLUSIONES}

- La agencia personal incrementa en el grupo de beneficiarios del programa Juntos que lleva de 3 años a más. Es decir que el programa Juntos promueve esta capacidad en las beneficiarias.

- El nivel de empoderamiento no encuentra diferencia significativa entre ambos grupos. Debido a que existen factores externos preexistentes que aún dificultan su desarrollo.

- La capacidad de agencia que logra desarrollarse a través del programa Juntos no consigue llegar a su punto máximo que es el empoderamiento; lo que conlleva a pensar que de suspenderse el programa Juntos, es 
probable que las beneficiarias omitan las conductas positivas hasta ahora logradas.

Ante los resultados encontrados, se señalan las siguientes recomendaciones:

- Es apropiado sugerir el desarrollo de actividades y programas que promuevan el desarrollo de las variables de estudio.

- Se sugiere mejorar los instrumentos de evaluación utilizados en el presente estudio y adecuarlos a la población rural en su propia lengua a fin de obtener mejores resultados.

- Es necesario realizar más estudios de investigación en el Perú en torno a la capacidad de agencia.

- En lo metodológico, se sugiere replicar la investigación a nivel nacional donde se tenga el programa Juntos y tal vez otros programas de corte social, que permitan la generalización de los resultados.

\section{REFERENCIAS}

Barbier, L. (2014). Agencia Personal y Bienestar subjetivo en mujeres Privadas de su libertad (Tesis de Licenciatura). Pontificia Universidad Católica del Perú.

Cohen, J. (1988). Statistical power analysis for the beh07avioral sciences (2nd Ed.). en Nolan, S. \& Heinzen, Th. (2011). Statistics for the Behavioral Sciences. USA: Worth Publichers.

Hernández, R.; Fernández, C. y Baptista, P. (2010). Metodología de la Investigación. Barcelona, España: Mc Graw Hill Interamericana.

Hogan, Th. (2004). Pruebas psicológicas. México: Manual Moderno.

Instituto de Estudios Peruano. (2009). "Estudio de percepción sobre cambios de comportamiento de los beneficiarios y accesibilidad al programa juntos en el distrito san Jerónimo (Andahuaylas -Apurímac). Recuperado de http://www.juntos.gob.pe.

Kerlinger, Fr. \& Lee, H. (2002). Investigación del comportamiento (4a Ed.). México: McGraw-Hill

Matijasevic, M.; Ramírez, M. y Villada, C. (2010). Felicidad, bienestar y capacidad de agencia. El caso de Manizales. Región Es, 5(1), 104 - 150. Recuperado de http:// www.ocaribe.org/cargar_imagen.phpid=126\&tipo=14\&thumbnail=FALSE.

Padilla, M. (2011). Agencia Personal y Estereotipos de Roles de Género en un Grupo de Mujeres Microempresarias.. (Tesis de Licenciatura). PUCP, Lima, Perú

Perova, E. y Vakis, R. (2011). Más tiempo en el programa, mejores resultados: duración e impactos del programa JUNTOS en el Perú. Recuperado de http://www.juntos. gob.pe. 
Pick, S. Sirkin, J., Ortega, I., Osorio, P., Martínez, R., Xocolotzin U. y Givaudan, M. (2007). Escala Para Medir Agencia Personal y Empoderamiento (ESAGE). Revista Interamericana de Psicología, 41(3), 295-304. Recuperado de http:/www.psicorip. org/Resumos/PerP/RIP/RIP041a5/RIP04131.pdf.

Pick, S., García, R. G. \& Leenen, I. (2011). Modelo para la promoción de la salud en comunidades rurales a través del desarrollo de agencia personal y empoderamiento intrínseco. Universitas Psychologica, 10(2), 327-340. Recuperado de http://revistas. javeriana.edu.co/index.php/revPsycho/article/view/510/1076.

Valladares, L. (2011). Autonomía, agencia y empoderamiento:El papel de las prácticas interculturales. XII Congreso Internacional de Teoría de la Educación. Recuperado de http://www.cite2011.com. 